\title{
CONSERVATION DES GAMĖTES DE TRUITES ARC-EN-CIEL ET FARIO EN CONDITIONS

\author{
in vitro ET post mortem
}

\author{
Jacqueline MARCEL (1), R. BILLARD, D. MATEI (2) \\ Laboratoire de Physiologie des Poissons \\ I.N.R.A., 78350 JOUY EN JOSAS, France
}

\section{RÉSUMÉ}

Une expérimentation a été conduite dans le but de tester la durée de survie des gamètes laissés dans le corps de géniteurs morts stockés à $4^{\circ} \mathrm{C}$. Un témoin est constitué d'une part par des gamètes fraîchement prélevés et d'autre part par des gamètes prélevés avant sacrifice des géniteurs et conservés in vitro à $4^{\circ} \mathrm{C}$.

Le pouvoir fécondant du sperme laissé dans le canal déférent de mâles morts ne se maintient que quelques heures et diminue dès la 6ème heure post mortem, et cela dans les deux espèces testées. Par contre, le sperme provenant des mêmes mâles et conservé in vitro conserve son pouvoir fécondant pendant une durée de 60 à $120 \mathrm{~h}$ chez la truite Arc-en-ciel et $14 \mathrm{j}$. chez la truite Fario. Le sperme fraîchement prélevé sur des mâles vivants conserve un pouvoir fécondant élevé pendant toute la durée de l'expérience.

La fécondabilité des ovules ne se maintient que 3 à $6 \mathrm{~h}$ chez la truite Arc-en-ciel et $12 \mathrm{~h}$ chez la truite Fario. Les ovules témoin in vitro ne se conservent que quelques heures chiez la truite Arc-en-ciel et au moins $72 \mathrm{~h}$ chez la truite Fario.

La conservation en condition post mortem est donc plus défavorable à la survie des gamètes que la conservation in vitro.

\section{SUMMARY}

Rainbow trout and brown trout gamete storage in in vitro and post mortem conditions.

The survival time of gametes left in the body of dead brood fish stocked at $4^{\circ} \mathrm{C}$ was tested. The control was: (i).fresh gametes and (ii) gametes taken before the sacrifice of brood fish and stored in vitro at $4^{\circ} \mathrm{C}$.

The sperm left in the vas deferens of dead males only retained its fertilizing ability for several hours and decreased from hour 6 post mortem in both the species tested. On the other hand, the sperm of the same males stored in vitro kept its fertilizing ability for 60 to $120 \mathrm{hrs}$ in rainbow trout and 14 days in brown trout. The fertilizing ability of fresh sperm from live males remained high during the whole experiment.

The fertilizability of ova was only maintained from 3 to 6 hrs in rainbow trout and 12 hrs in brown trout. The control ova of rainbow trout survived only several hrs in vitro and those of brown trout less than 72 hrs.

Storage post mortem is less favorable to gamete survival than in vitro storage.

\section{INTRODUCTION}

La survie des gamètes de salmonidés laissés dans le tractus génital ou la cavité générale après la mort des géniteurs n'a pas fait l'objet d'investigations poussées. SMITH et QUISTORFF (1943), OKADA et al. (1956) ont abordé ce problème et montré que le pouvoir fécondant du sperme de saumon chinook diminuait en quelques heures après la mort du mâle. Chez la femelle des survies d'ovules en condition post mortem de $8 \mathrm{~h}$ ont été rapportées par OKADA è al. (1956) chez le saumon Oncorhynchus keta alors que KAZAKOV (1979) trouve une décroissance de la fertilité dès la 2ème heure suivant la mort de l'animal chez le saumon (Salmo salar). Cette question a été reprise dans la présente expérience où des gamètes ont été conservés dans des animaux morts et in vitro.chez la

(1) Élève de l'EPHE, Laborațoire d'étude du développement post-embryonnaire des vertébrés inférieurs, 25, rue du Plat, 69288 LYON Cédex 1.

(2) Adresse actuelle: Statiunea de cercetari piscicole, Podu lloaiei Podu Iloaiei, 6623 IASI, Roumanie. 
truite Arc-en-ciel et la truite Fario. Une partie de ces résultats a été présentée par ailleurs (MATEl et al., 1980; BILLARD et al., 1980).

\section{MATÉRIEL ET MÉTHODES}

Les géniteurs de truite Arc-en-ciel (Salmo gairdneri)(souche Lubin) et Fario/Salmo trutta fario) (souche Vermenoux), tous âgés de $3 \mathrm{ans}^{+}$et pesant entre 500 et $1500 \mathrm{~g}$, sont élevés dans la pisciculture de Gournay sur Aronde et transportés au laboratoire après ovulation et entrée en spermiation. L'expérimentation débute le 4ème jour suivant l'arrivée des géniteurs au laboratoire. Les femelles ont alors ovulé depuis moins de $8 \mathrm{j}$. et les mâles sont entrés en spermiation depuis 2 à 3 semaines. Les expériences se sont déroulées successivement en novembre 1979 pour la truite Arc-en-ciel et en novembredécembre pour la truite Fario.

Dans toutes les expériences, les géniteurs ont été groupés par lots de 6 à 10 individus : un lot est conservé comme "témoin in vivo", dans un autre lot une partie des gamètes est prélevée et stockée à $+4^{\circ} \mathrm{C}$ au réfrigérateur, une autre est laissée dans les animaux qui sont sacrifiés (assommés) et stockés à $+4^{\circ} \mathrm{C}$.

Les gamètes conservés in vitro sont maintenus à l'obscurité, les ovules sont laissés dans leur liquide cœlomique et conservés par lot de 200 environ dans des coupelles. Le sperme est conservé en piluliers de $20 \mathrm{ml}$ laissés ouverts et la hauteur de sperme dans le pilulier est de $0,5 \mathrm{~cm}$; avant chaque prélèvement le flacon est agité.

Les ovules et les spermatozoïdes conservés in vitro ou laissés dans les géniteurs sacrifiés sont prélevés à des temps croissants après le début de l'expérience et sont inséminés avec des gamètes fraîchement prélevés sur les lots témoin in vivo, mâle et femelle. Les ovules conservés sont donc inséminés avec du sperme frais et inversement. L'insémination pratiquée selon BILLARD (1977) porte sur 200 ovules, $10 \mathrm{ml}$ de dilueur et $10 \mu \mathrm{l}$ de sperme (dilution 103); après un séjour de $15 \mathrm{~min}$. dans le milieu d'insémination, les ovules sont transférés en eau douce où l'incubation est conduite pendant environ $20 \mathrm{j}$. à $10^{\circ} \mathrm{C}$. Les œufs sont alors fixés dans le liquide de Stockard et le dénombrement porte sur les œufs embryonnés normaux et anormaux et les œufs non embryonnés.

Du fait de la forte variabilité des résultats, l'évolution de la fertilité des gamètes exprimée par le pourcentage d'œufs embryonnés est présentée individuellement sur les graphiques pour chaque individu. Ce pourcentage sera considéré comme l'expression du pouvoir fécondant du sperme ou l'aptitude a la fécondation des ovules. Les résultats sont comparés par test $t$ après transformation angulaire des données.

\section{RÉSULTATS}

\section{- Evolution du pouvoir fécondant du sperme}

Chez la truite Arc-en-ciel le pouvoir fécondant des mâles témoin (sperme témoin in vivo) reste voisin de $90-95 \%$ pendant la période expérimentale de $150 \mathrm{~h}$, malgré quelques fluctuations dues vraisemblablement à la qualité des ovules (Fig. 1). Dans le lot expérimental, les spermes conservés in vitro conservent leur pouvoir fécondant initial pendant des durées variant entre 54 et $126 \mathrm{~h}$ selon les mâles (Fig. 2). Après $126 \mathrm{~h}$ les taux moyens de fécondation sont significativement plus faibles $(P<0,01)$ que ceux des témoins in vivo. En condition post mortem, ces mêmes spermes ne survivent que quelques heures; à $6 \mathrm{~h}$, le pouvoir fécondant a déjà diminué et se trouve nul à $12 \mathrm{~h}$ (Fig. 3).

Chez la truite Fario, on retrouve les mêmes résultats. II n'y a pas de changement dans le pouvoir fécondant du sperme fraîchement récolté dans le lot "témoin in vivo" pendant une période de $14 \mathrm{j}$. (Fig. 4). Le sperme conservé in vitro conserve son pouvoir fécondant pendant $14 \mathrm{j}$. alors que, s'il est laissé dans le tractus génital de mâles morts, le pouvoir fécondant devient pratiquement nul en l'espace de $18 \mathrm{~h}$ (Fig. 4). Par rapport au témoin in vivo ou aux ceufs conservés in vitro, la chute du taux d'embryonnement devient significative dès la 12 ème heure $(P<0,05)$.

\section{- Evolution de l'aptitude des ovules à la fécondation}

Chez la truite Arc-en-ciel, la chute de fécondabilité des ovules conservés in vitro et post mortem est rapide. Elle devient nulle après $12-18 \mathrm{~h}$ en condition post-mortem et n'est que d'environ $20 \%$ après $36 \mathrm{~h}$ de conservation in vitro. La qualité s'altère plus rapidement post mortem qu' in vitro ( $\mathrm{P}<0,001$ à la 12 ème heure) (Fig. 5). La fécondabilité des ovules témoin in vivo périodiquement prélevés sur le lot de femelles vivantes est 


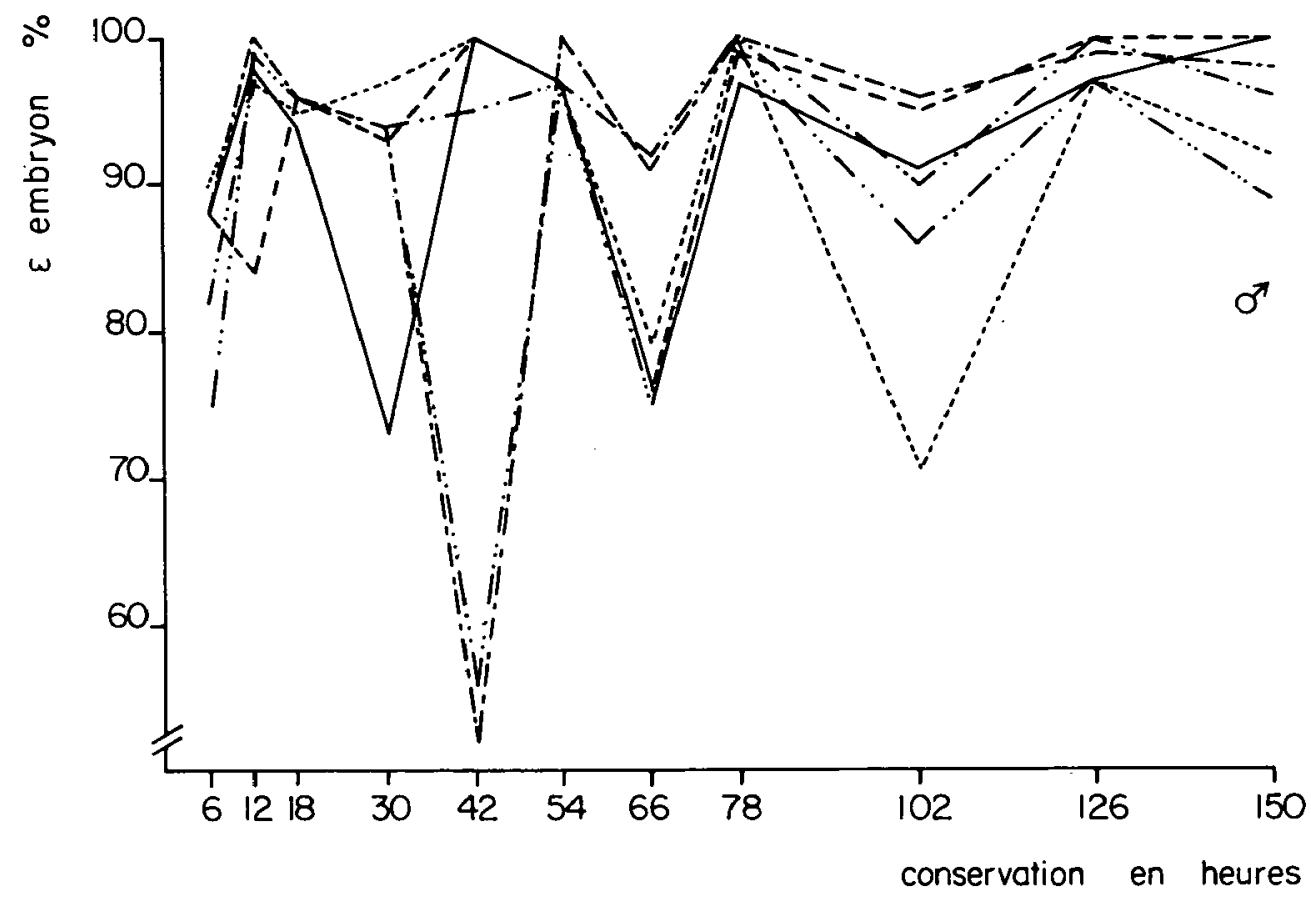

Figure 1 : Evolution du pouvoir fécondant du sperme prélevé périodiquement pendant une période de $150 \mathrm{~h}$ sur des mâles vivants ; truite Arc-enciel.

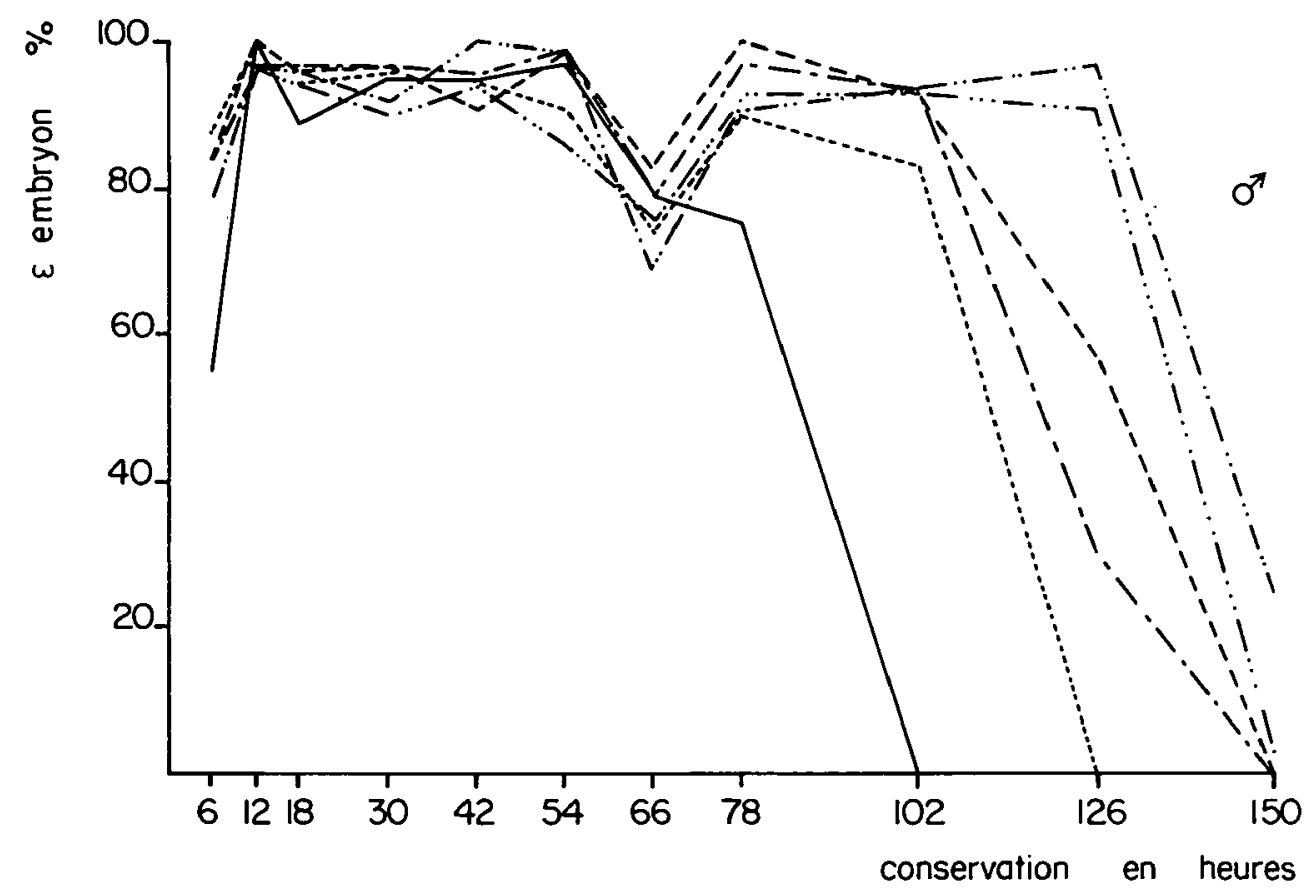

Figure 2: Pouvoir fécondant de spermes conservés in vitro pendant $150 \mathrm{~h}$; truite Arc-en-ciel. 
restée relativement stable pendant la période expérimentale de $36 \mathrm{~h}$ (données non présentées).

Chez la truite Fario, la fécondabilité des ovules post mortem diminue plus rapidement que celle des ovules in vitro ( $\mathrm{P}<0,001$ à $24 \mathrm{~h}$ ) (Fig. 6). Pour ces derniers, on observe une légère chute de l'ordre de $10 \%$ entre 0 et $18 \mathrm{~h}$ puis le niveau de fertilité se stabilise jusqu'à la fin de l'expérience à $72 \mathrm{~h}$. La fécondabilité des ovules témoin in vivo ne varie pas au cours de l'expérience et reste voisine de $95 \%$ (Fig. 6).

\section{Discussion}

La survie des gamètes de truite apparait donc extrêmement brève (quelques heures) lorsqu'ils séjournent dans le tractus génital ou la cavité cœlomique de géniteurs morts; les possibilités de récupérer des gamètes sur des animaux morts, dans le cas d'accident par exemple, sont donc très limitées. Par contre, la survie des gamètes conservés in vitro est plus longue, en particulier pour le sperme. Ainsi si l'on désire transférer des gamètes, il est préférable de les extraire des géniteurs plutôt que de transporter ces derniers si les risques de mortalité sont grands.

La chute rapide de la fertilité des gamètes en situation post mortem semble pouvoir être rapprochée des conditions d'anoxie qui se sont révélées très défavorables à la sur vie des spermatozoïdes (TRUSCOTT et al., 1968 et STOSS et al., 1978; BILLARD, 1980). De même, le stockage d'ovules de truite en sacs gonflés d'oxygène améliore leur durée de conservation et le niveau de fertilité (BILLARD et LEGENDRE, 1982). Il est vraisemblable que les différences dans les résuitats de cette expérience et celles de SMITH et QUISTROFF (1943) soient dues aux conditions d'oxygénation. En effet, ces auteurs trouvaient comme NAKANO et NOZAWA (1925) que le pouvoir fécondant du sperme de saumon chinook n'était modifié qu'après un séjour de $5 \mathrm{~h}$ dans des mâles morts alors que ce même sperme. prélevé avant la mort des animaux, et transporté en flacons bouchés et entièrement remplis présentait des chutes de pouvoir fécondant de $10 \%$ après $2 \mathrm{~h} 30$ et $46 \%$ après $5 \mathrm{~h}$. On sait que dans ces conditions, les spermatozoïdes se trouvent rapidement en anoxie (STOSS et al., 1978; LEGENDRE et BILLARD, 1980). En effet, la consommation d' $\mathrm{O}_{2}$ par le sperme est élevée $\left(21 \mathrm{~mm}^{3} / \mathrm{h} / \mathrm{ml}\right.$ à $\left.10^{\circ} \mathrm{C}\right)$ (OKADA et al., 1956; SCOTT et BAYNES, 1980 pour revue).

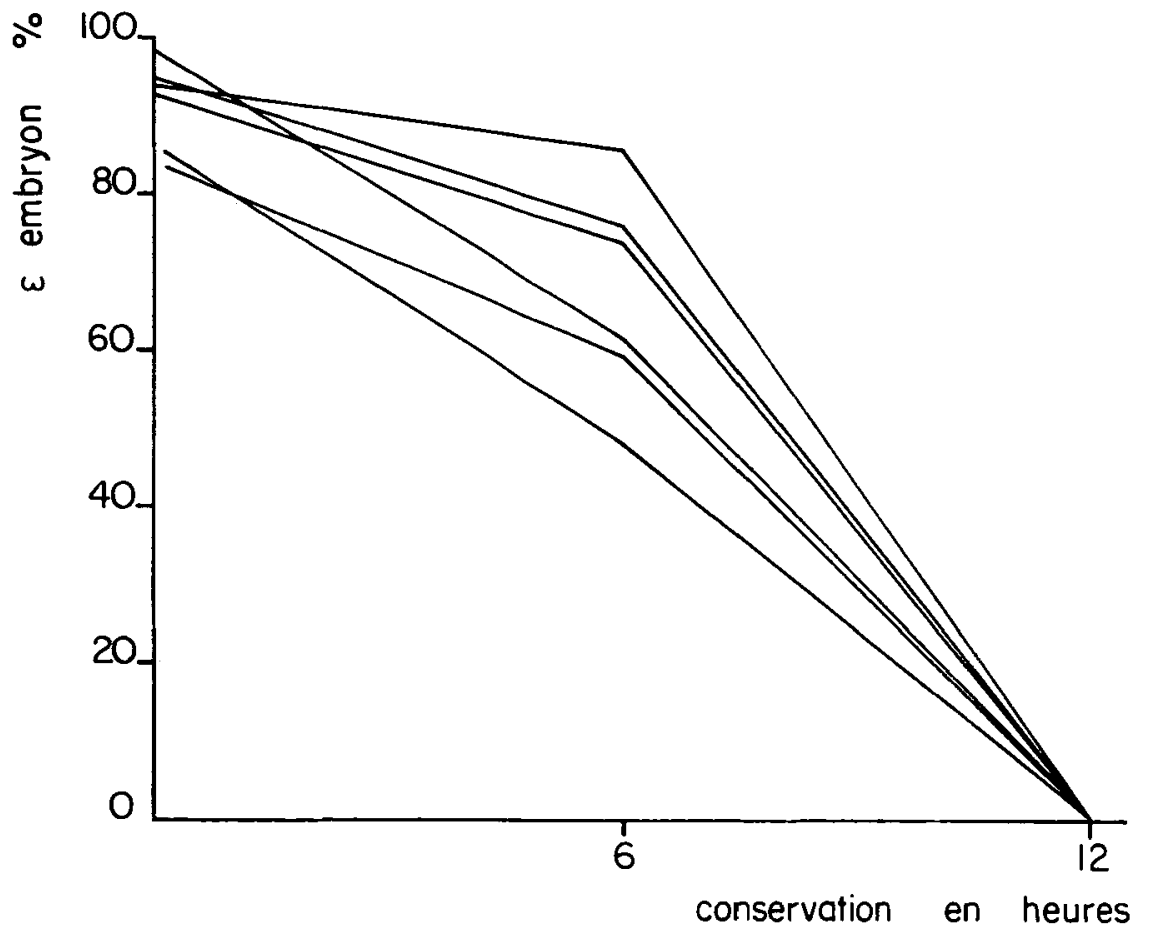

Figure 3 : Pouvoir fécondant des mêmes spermes que sur la fig. 2, mais laissés dans le canal déférent des mâles après sacrifice et stockage à $4^{\circ} \mathrm{C}$ à "sec"; truite Arc-en-ciel. 

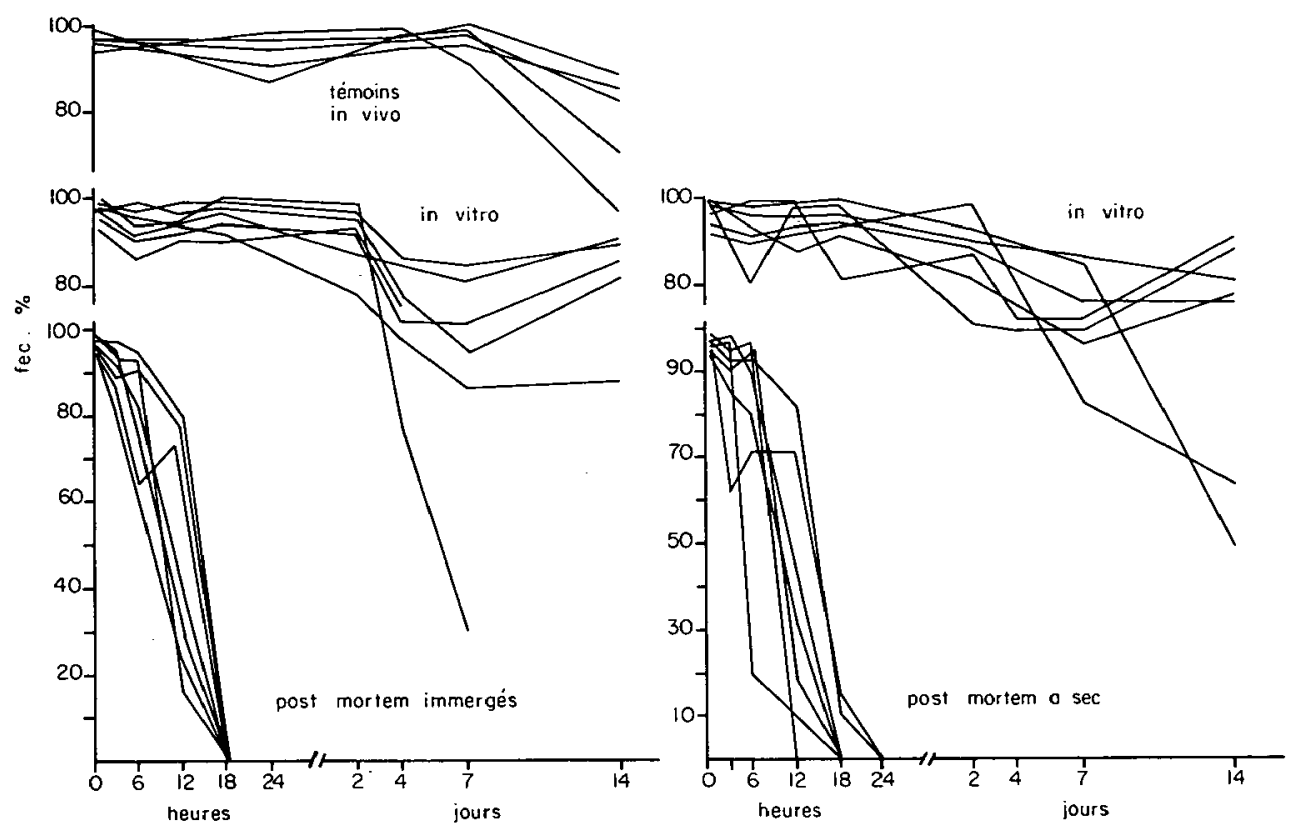

Figure 4 : Pouvoir fécondant de sparmes de truite Fario. Lot témoin in vivo: sperme fraîchement prélevé pendant une période de 14 jours: spermes conservés in vitro à $4^{\circ} \mathrm{C}$, et spermes laissés dans le canal déférent de mâles après sacrifice (post mortem).

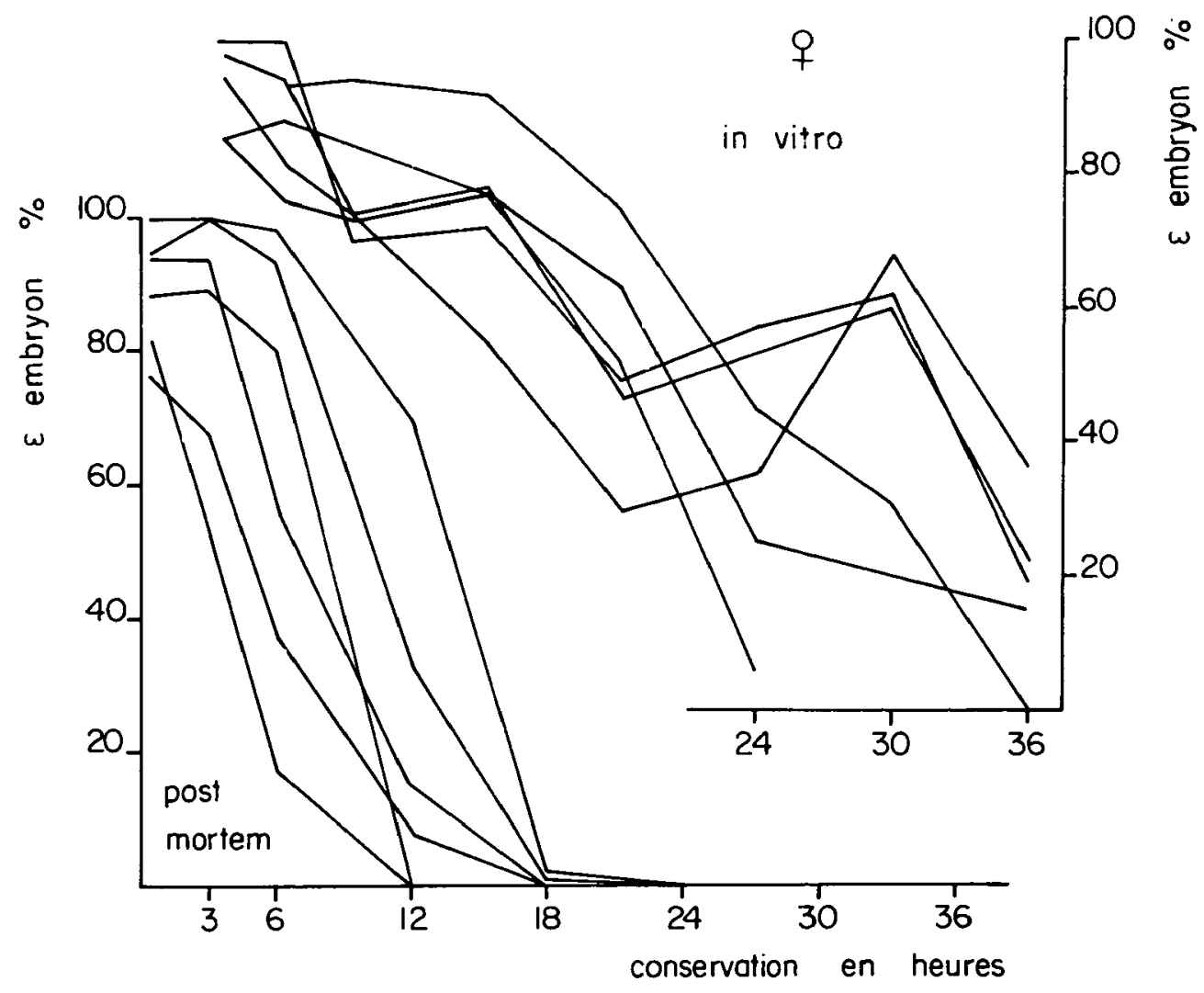

Figure 5 : Evolution de l'aptitude à la fécondation d'ovules de truite Arc-en-ciel conservés in vitro à $4^{\circ} \mathrm{C}$ ou laissés dans la cavité générale après sacrifice des femelles (post mortem). 
Les possibilités de conservation in vitro sont intéressantes d'un point de vue pratique. La durée de conservation du sperme dans ces conditions est plus longue que celle des ovules. On peut mettre à profit ces possibilités pour simplifier les opérations d'insémination artificielle et, comme l'ont proposé LEGENDRE et BILLARD. (1980), prélever le sperme d'abord puis les ovules, ce qui évite la manipulation simultanée des mâles et des femelles. Cependant, il faut tenir compte de la forte variabilité individuelle de la conservation des pouvoirs fécondants (cf. Fig. 2) et du fait qu'il n'existe pas de critères d'appréciation fiables (CARPENTIER et BILLARD, 1978). D'autre part, les résultats ne peuvent être généralisés à l'ensemble des salmonidés et la présente expérience montre qu'il existe des grandes différences entre Arc-en-ciel et Fario, la survie étant meilleure chez la truite Fario que chez la truite Arc-en-ciel, à la fois pour les spermatozoïdes et les ovules, alors que les conditions d'élevage des géniteurs et de recueil des gamètes sont les mêmes dans les deux cas. Cependant CYKOWSKA et al. (1973) avancent que la durée de survie des ovules conservés in vitro est de $12 \mathrm{~h}$ pour la truite Fario et $24 \mathrm{~h}$ pour la truite Arc-en-ciel, mais on ignore si les ovules des espèces étaient comparables tant du point de vue maintenance des géniteurs que temps écoulé entre ovulation et insémination. Même à l'intérieur d'une même espèce comme la truite Arc-en-ciel, les données bibliographiques nous montrent qu'il existe de très fortes variabilités dans la qualité des gamètes. Par exemple, dans cette expérience, le maintien in vitro des ovules de truite Arc-en-ciel sans perte de fertilité ne dépasse pas $3 \mathrm{~h}$, ce qui semble être une valeur faible par rapport aux autres données de la littérature qui sont généralement de l'ordre de $24 \mathrm{~h}$ ou plus: GINSBURG, 1968 ; WITHLER et MORLEY, 1968 ; TAKANO et al., 1973 ; CARPENTIER et BILLARD, 1978.

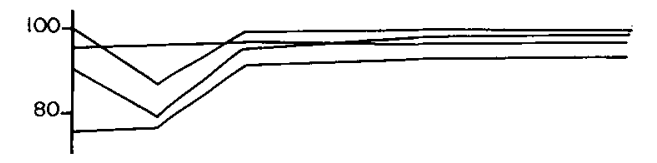

témoin in vivo
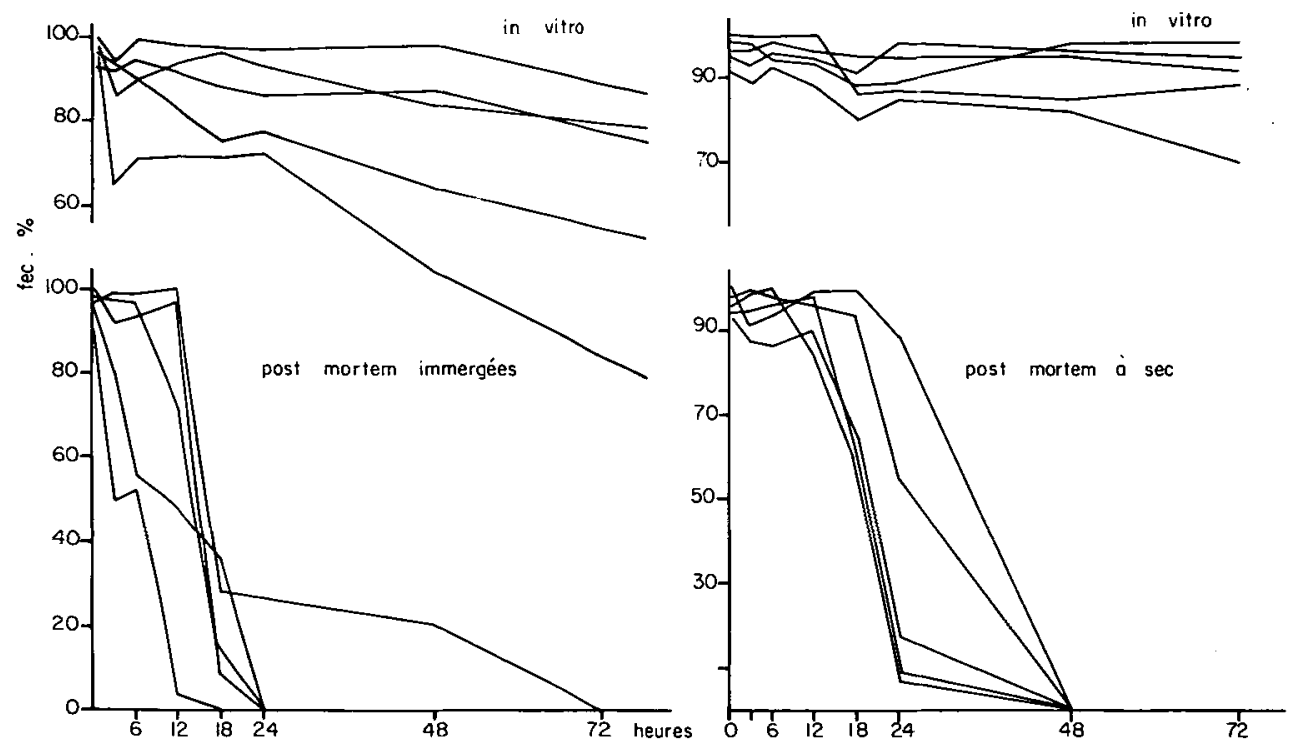

Figure 6 : Evolution de l'aptitude à la fécondation d'ovules de truite Fario conservés in vitro ou laissés dans la cavité générale après sacrifice des femelles (post mortem).

Il faut aussi insister sur le fait que dans la présente expérience, les ovules des deux espèces réagissent très différemment à la conservation in vitro et même en situation post mortem par rapport à la conservation in vivo où les niveaux de fertilité sont intégralement conservés pendant la durée de l'expérimentation. On sait d'autre part que la conservation in vivo post ovulation peut se poursuivre pendant plusieurs semaines (ESCAFFRE et al., 1977). II y a donc lieu de rechercher plus avant les conditions de conservation in vivo afin de les reproduire et de se doter de techniques de conservation fiables fort utiles dans la pratique piscicole et dans la gestion des populations. 


\section{REMERCIEMENTS}

Ce travail a été réalisé dans le cadre d'une convention de recherche CSP-INRA. Le séjour de M. D. MATEl en France s'est effectué dans le cadre des échanges de coopération scientifique Franco-Roumains.

\section{RÉFÉRENCES BIBLIOGRAPHIQUES}

BILLARD R., 1977. Utilisation d'un système Tris-glycocolle pour tamponner le dilueur d'insémination de truite. Bull. Fr. Pisc., 264, $102-112$.

BILLARD R., 1980. Short term preservation of sperm under oxygen atmosphere in rainbow trout (Salmo gairdneri) Aquaculture, 23, 287-293.

BILLARD R., MARCEL J., MATEI D., 1980. Survie post mortem des gamètes de truite fario Salmo trutta fario. Can. J. Zool., 59, 29-33.

BILLARD R., LEGENDRE M., 1982. Conservation à court terme des qamètes de truite arcen-ciel en condition in vitro sous atmosphère d'oxygène. Bull. Fr. Pisc., 284, 162-167.

CARPENTIER M., BILLARD R., 1978. Conservation à court terme des gamètes de salmonidés à des températures voisines de $0^{\circ} \mathrm{C}$. Ann. Biol. anim. Bioch. Biophys. 18, 1083-1088.

CYKOWSKA C., SOBOCINSKI A., TOMASIK L., WINNICKI A., 1973. Studies on the salmonid eggs fertilization delayed in relation to the spawning. Zeszyty Naukome Akademii Rolniczejw Szczecinie, 40, 180-183.

ESCAFFRE A.M., PETIT J., BILLARD R., 1977. Evolution de la quantité d'ovules récoltés et conservation de leur aptitude à être fécondés à des températures voisines de $0^{\circ} \mathrm{C}$. Bull. Fr. Pisc., 265, 134-142.

GINSBURG A.S., 1968. Fertilization in fishes and the problem of polyspermy: Publishing House "Nauka", 351 pp.

KAZAKOV R.V., 1979. Storing of sexual products of atlantic salmon at low temperature. 3-19 in Qualité des reproducteurs et des produits sexuels des poissons Salmonidae (Coregones et Cyprinidae), Gosniork, Leningrad.

LEGENDRE M., BILLARD R., 1980. Cryoconservation du sperme de truite Arc-en-ciel (Salmo gairdneri R.) Bull. Fr. Pisc., 278, 11-33.

MATEI D., MARCEL J., BILLARD R., 1980. Survie des gamètes en conditions in vitro et post mortem chez la truite arc-en-ciel. Bull. Acad. Sci. Agr. For., 10, 211-218.

NAKANO S., NOZAWA A., 1925. On the vitality of the eggs and sperm of Oncorhynchus masou (land-locked). J. Imp. Fisher, Institute Vol. XXI, 8, 17.

OKADA S., ISHIKAWA Y., KIMURA G., 1956. On the viability of the sperm and the egg left in the dead body of Dog-Salmon, Oncorhynchus keta (Walbaum). Scient. Rep. Hokkaido. Fish Hatch, 11, 7-18.

SMITH R.T., QUISTORFF E., 1943. Experiments with the spermatozoa of the steelhead trout, Salmo gairdneri and the chinook salmon Oncorhynchus tschawytscha. Copeia, 3, 164-167.

SCOTT A.P. and BAYNES S.M., 1980. A review of the biology, handling and storage of salmonid spermatozoa. J. Fish Biol., 17, 707-739.

STOSS J., BUYYUKKHATIPOGLU S., HOLTZW., 1978. Short term and cryopreservation of rainbow trout (Salmo gairdneri Richardson) sperm. Ann. Biol. anim. Bioch: Biophys., 18, 1077-1082.

TAKANO K., HIROI O., YASUKAWA M., SHETAKE T., 1973. Studies on the retention of gametes of salmonid fishes. I- On the fertility of chum salmon eggs after storage. Sci. Rep. Hokkaido Salmon Hatchery, 27, 31-37.

TRUSCOTT B., IDLER D.R., HOYLE R.J., FREEMAN H.C., 1968. Sub-zero preservation of atlantic salmon sperm. J. Fish. Res. Bd. Can., 25, 363-372.

WITHLER F.C., MORLEY R.B., 1968. Effects of chilled storage on viability of stored ova and sperm of sockeye and pink salmon. J. Fish. Res. Bd. Can., 25, 2695-2699. 